\title{
Analysis Method of Sucker Rod Centralizer Spacing in Three-dimensional Hole
}

\author{
Tao Ren ${ }^{\mathrm{a}}$, Zhenjiang Nan, Wen Sun ${ }^{\mathrm{b}}$, and Xiaoqing Kang \\ School of Mechanical Engineering, Xi'an Shiyou University, Shaanxi, Xi'an, 710065, P.R.China \\ arentao365@126.com, bsunwen@xsyu.edu.cn.
}

Keywords: Centralizer; Eccentric wear of rods and tubing; Optimization design; Three-dimensional hole

\begin{abstract}
The usage rate of sucker rod centralizer more and more broad with the development of a sucker rod pump, adding sucker rod centralizer is very effective to reduce the impact of rod and tubing wear, increase the sucker rod and tubing service life, reduce the frequency of maintenance and the production cost .Starting from the actual borehole parameters, comprehensively considering bending stress condition of sucker rod string in the three-dimensional hole, analyzing of the axial load of sucker rod and lateral loads, and use the lateral deformation of the sucker rod equal annular space between tubing and sucker rod as the basic principle of centralizer allocation, to calculate and analysis of the spacing configuration of the stabilizer. The example analyzed shows that optimization design of sucker rod centralizer spacing has a certain reference value.
\end{abstract}

\section{Introduction}

Sucker rod pumping system is one of the most widely used oil pumping methods at home and abroad. The friction and wear between sucker rod and tubing is always an important issue needs resolution during the rod pumping system works steadily,It will cause sorts of downhole accidents indirectly, result in oil well stop production, increase oil well maintenance times and repair costs and directly affect the oil well production benefit[1-3]. In view of the eccentric wear of the rod tube, oil workers at home and abroad have made a lot of theoretical research, and achieved certain results. various measures for preventing side wear have emerged. The installation of the stabilizer on the sucker rod string is a widely accepted method for oil field, which has low cost and good effect. Sucker rod centralizer is very effective to reduce the impact of rod and tubing wear,increase the sucker rod and tubing service life and reduce the frequency of maintenance and the production cost.

At present ,the optimum locating positions and intervals of the rod centralizers lacking of support theory to guide and carry out concrete basis, most wells are configured by experience to design the locating positions and intervals of the rod centralizers, in addition to observing wear during the workover, to position centralizer at badly worn. This paper from the actual borehole parameters, comprehensively considerating bending stress condition of sucker rod string in the three-dimensional hole, analyzing of the axial load of sucker rod and lateral loads, and use the lateral deformation of the sucker rod equal annular space between tubing and sucker rod as the basic principle of centralizer allocation, to calculate and analysis of the spacing configuration of the stabilizer.

\section{Axial load of a sucker rod}

Axial force at the bottom of a sucker rod. A sucker rod is limited at the bottom by a variety of load included the force acting on the plunger by the weight of the liquid $W_{L}$, the force produced by liquid inertia $W_{L a u}$, the buoyancy of well liquid and the sucker-rod string $W_{f}$, the friction between the liquid column and the tubing $W_{t L}$, the friction force between the piston and the bush $W_{b p}$ and the friction force of well fluid through the subsurface pump $W_{p L}$ during the up and down stroke. 
Axial force at the bottom of a sucker rod during the up stroke $W_{u b}$

$$
W_{u b}=W_{L}+W_{L a u}+W_{t L}+W_{b p}
$$

Axial force at the bottom of a sucker rod during the down stroke $W_{d b}$

$$
W_{d b}=W_{f}+W_{b p}+W_{p L}
$$

Axial force on top of a segment of a sucker rod between two centralizers. Assuming that the rod string centralizer has been installed, calculating the axial force on top of a segment of a sucker rod between two centralizers. The axial force of the segment rod string included the segment rod string dead weight $W_{r d}$, inertial load $W_{a d}$, the buoyancy of the segment rod string $W_{f d}$ and the friction force between the segment rod string and liquid column $W_{r L}$. Due to the load of sucker rod string is not the same during the up and down stroke, so the axial force on top of a segment of a sucker rod between two centralizers is calculated according to the up and down stroke.

The unit load of a segment of a sucker rod between two centralizers during the up stroke $q_{u d}$ is

$$
q_{u d}=\left(W_{r d}+W_{a d}-W_{f d}\right) / l
$$

Axial force on top of a segment of a sucker rod between two centralizers during the up stroke $W_{u s}$ is:

$$
W_{u s}=W_{x d}+q_{u d} l=W_{x d}+\left(W_{r d}+W_{a d}-W_{f d}\right) / l
$$

Where: $W_{x d}$ is axial force at the end of a segment of a sucker rod, at the first cross axial force is $W_{u b}$.

The unit load of a segment of a sucker rod between two centralizers during the down stroke $q_{d d}$ is:

$$
q_{d d}=\left(W_{r d}-W_{a d}-W_{f d}-W_{r L}\right) / l
$$

Axial force on top of a segment of a sucker rod between two centralizers during the down stroke $W_{d s}$ is:

$$
W_{d s}=W_{x d}-q_{d d} l=W_{x d}-\left(W_{r d}-W_{a d}-W_{f d}-W_{r L}\right)
$$

Where: $W_{x d}$ is axial force at the end of a segment of a sucker rod, at the first cross axial force is $W_{d b}$.

\section{Horizontal load of a sucker rod}

Fig.1 shows the three-dimensional space curve of a sucker rod between two centralizers. Well the principal normal direction of hole is $n$ and the vice-normal direction of hole is $m$. The oblique plane where the principal normal direction of the surface is called the dogleg, oblique plane where the vice-normal direction of the surface is orthogonal with the dogleg plane, and corresponding $m$ is orthogonal with $n$.

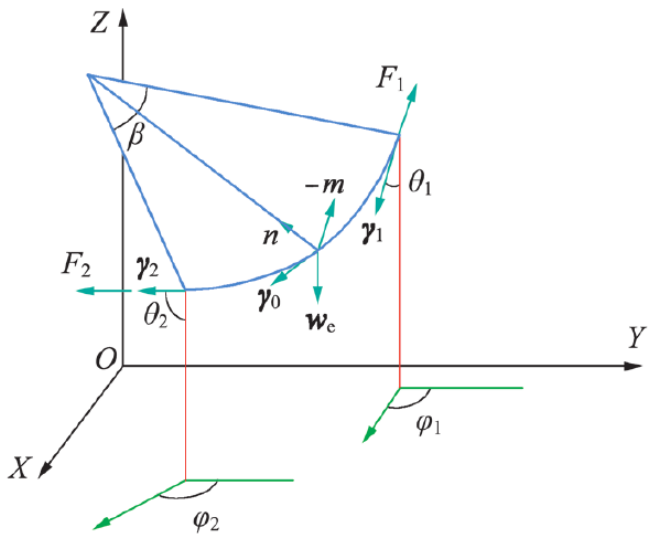

Fig.1 Three-dimensional space curve of a sucker rod

General variation angle. Given by the formula (Lu-binski) [4]

$$
\cos \beta=\cos \theta_{1} \cos \theta_{2}+\sin \theta_{1} \sin \theta_{2} \cos \left(\varphi_{2}-\varphi_{1}\right)
$$

Where: $\theta_{1} 、 \theta_{2} 、 \varphi_{1}, \varphi_{2}$ is respectively the well angle and azimuth angle of the two end points of 
sucker rod between two centralizers in three dimensional well eyes.

Vice-normal vector of hole section $m$ is [3]

$$
m=m_{1} i+m_{2} j+m_{3} k
$$

Where:

$$
\left\{\begin{array}{l}
m_{1}=\sin \theta_{2} \cos \varphi_{2} \cos \theta_{1}-\sin \theta_{1} \cos \varphi_{1} \cos \theta_{2} \\
m_{2}=\sin \theta_{1} \sin \varphi_{1} \cos \theta_{2}-\sin \theta_{2} \cos \varphi_{2} \cos \theta_{1} \\
m_{3}=\sin \theta_{1} \sin \varphi_{1} \sin \theta_{2} \cos \varphi_{2}-\sin \theta_{2} \sin \varphi_{2} \sin \theta_{1} \cos \varphi_{1}
\end{array}\right.
$$

Principal normal vector of hole section $n$ is [3]

$$
n=\left(m_{2} t_{3}-m_{3} t_{2}\right) i+\left(m_{3} t_{1}-m_{1} t_{3}\right) j+\left(m_{1} t_{2}-m_{2} t_{1}\right) k
$$

Where:

$$
\left\{\begin{array}{l}
t_{1}=\sin \theta_{1} \sin \varphi_{1}+\sin \theta_{2} \sin \varphi_{2} \\
t_{2}=\sin \theta_{1} \cos \varphi_{1}+\sin \theta_{2} \cos \varphi_{2} \\
m_{3}=\cos \theta_{1}+\cos \theta_{2}
\end{array}\right.
$$

Horizontal load in the direction of principal normal $F_{n l}$ is [3]

$$
F_{n l}=2 F_{2} \sin (\beta / 2)+W_{r d} l \sin \left(\left(\theta_{1}+\theta_{2}\right) / 2\right) \sin \left(\left(\theta_{1}-\theta_{2}\right) / 2\right) / \sin (\beta / 2)
$$

Horizontal load in the direction of vice-normal $F_{m l}$ is [3]

$$
F_{m l}=W_{r d} l \sin (\beta) \sin \left(\theta_{2}\right) \sin \left(\theta_{1}\right) \sin \left(\varphi_{2}-\varphi_{1}\right)
$$

In summary:horizontal load of a sucker rod between two centralizers $F_{H}$ is

$$
F_{H}=\sqrt{F_{n l}^{2}+F_{m l}^{2}}
$$

\section{Lateral deformation of a sucker rod between two centralizers}

In the three-dimensional well, lateral deformation of a sucker rod is produced by the axial tension and dead weight of a sucker rod. The calculation of the lateral deformation of a sucker rod in three dimensional wells is given by the following formula [5]

$$
\delta=\left(\frac{F_{H} l^{3}}{384 E I}\right)\left(\frac{24}{u^{4}}\right)\left(\frac{u^{2}}{2}-\frac{u \cosh u-u}{\sinh u}\right)
$$

Where: $u=\sqrt{\frac{F l^{2}}{4 E I}}, \mathrm{~F}$ is Axial tension of the segment rod string, $E$ is material elastic modulus; I is the cross-section moment of inertia of sucker rod.

\section{Calculation of two sucker rod centralizers spacing}

According to the maximum deformation of the sucker rod between the two stabilizer, the spacing of the sucker rod stabilizer is designed. Its design criteria are

$$
\varepsilon=\frac{f\left(d_{t}-d_{r}\right)}{2}
$$

Where: $f$ is the deformation ratio coefficient, $d_{i t}$ is the diameter of tube, $d_{r}$ is the diameter of sucker-rod.

When $\delta<\varepsilon$ increased $l$, then decreased $l$.Thus we can established one element nonlinear equation as following (17) and the numerical calculation method is used to solve the nonlinear equation.

$$
\left(\frac{F_{H} l^{3}}{384 E I}\right)\left(\frac{24}{u^{4}}\right)\left(\frac{u^{2}}{2}-\frac{u \cosh u-u}{\sinh u}\right)=\frac{f\left(d_{t}-d_{r}\right)}{2}
$$




\section{Application example}

A sucker-rod pumping pump depth is $1660 \mathrm{~m}$, submergence depth is $30 \mathrm{~m}$, plunger diameter is $22 \mathrm{~mm}$, length of stroke is $2.1 \mathrm{~m}$, pumping speed is $4 / \mathrm{min}$. Combination of sucker rod is $\varphi 16 \mathrm{~mm} \times 332 \mathrm{~m}+\varphi 19 \mathrm{~mm} \times 830 \mathrm{~m}+\varphi 22 \mathrm{~mm} \times 498 \mathrm{~m}$. The density of well liquid is $0.85 \mathrm{t} / \mathrm{m}^{3}$, dynamic viscosity of the well fluid is $0.065 \mathrm{pa}$.s , dimension of inner diameter of tubing is $63 \mathrm{~mm}$. Using the MATLAB software to optimize design of sucker rod centralizer spacing. Calculation results of centralizer spacing in Table 1 , Calculation results of centralizer location in Table 2, Arrangement of the centralizer in Fig.2.

Table 1 Calculation results of centralizer spacing

\begin{tabular}{ccccccccc}
\hline serial number & 1 & 2 & 3 & 4 & 5 & 6 & 7 & 8 \\
\hline $\mathrm{m}$ & 13.5 & 24.5 & 22.5 & 20.5 & 21 & 19 & 19 & 19.5 \\
\hline serial number & 9 & 10 & 11 & 12 & 13 & $\ldots$ & 45 & 46 \\
\hline $\mathrm{m}$ & 17 & 17 & 18 & 17 & 17.5 & $\ldots$ & 45 & 79 \\
\hline serial number & 47 & 48 & 49 & 50 & 51 & 52 & 53 & 54 \\
\hline $\mathrm{m}$ & 76 & 61 & 71.5 & 82.5 & 73.5 & 46 & 43 & 63.5 \\
\hline
\end{tabular}

Table 2 Calculation results of centralizer location

\begin{tabular}{ccccccccc}
\hline serial number & 1 & 2 & 3 & 4 & 5 & 6 & 7 & 8 \\
\hline $\mathrm{m}$ & 1646.5 & 1622 & 1599.5 & 1579 & 1558 & 1539 & 1520 & 1500.5 \\
\hline serial number & 9 & 10 & 11 & 12 & 13 & $\ldots$ & 45 & 46 \\
\hline $\mathrm{m}$ & 1483.5 & 1466.5 & 1448.5 & 1431.5 & 1414 & $\ldots$ & 776 & 697 \\
\hline serial number & 47 & 48 & 49 & 50 & 51 & 52 & 53 & 54 \\
\hline $\mathrm{m}$ & 621 & 560 & 488.5 & 406 & 332.5 & 286.5 & 243.5 & 180 \\
\hline
\end{tabular}

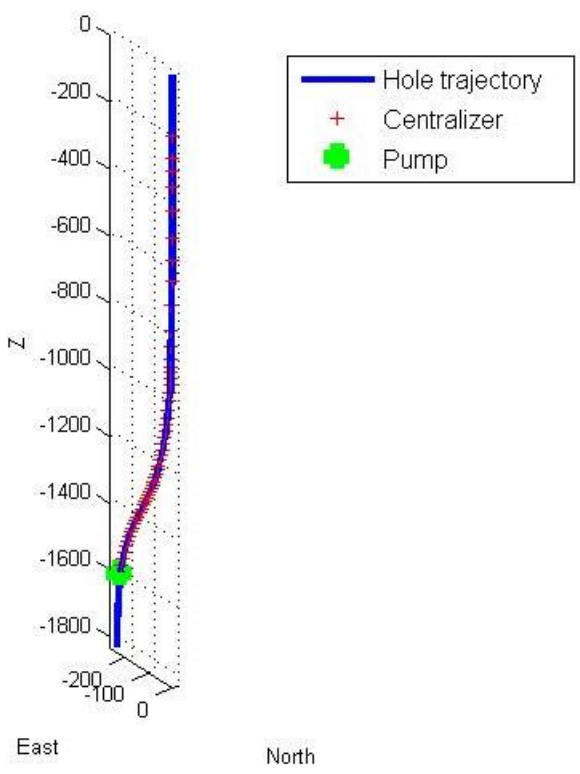

Fig.2 Arrangement of the centralizer

From the Table 1and the Table 2 we can figure out that there are 54 centralizers. The centralizer distribute closely because the force of sucker rod which at the end of a segment of a pump is larger and the trajectory curve of the borehole is larger. The upper centralizer distribute less because the force of sucker rod which at the top of a segment of a pump is little and the trajectory curve of the borehole is 
close to a straight line. Example analysis shows that the result of optimum design of sucker rod centralizer spacing has a certain reference value.

\section{Conclusions}

This paper from the actual borehole parameters, comprehensively considerating bending stress condition of sucker rod string in the three-dimensional hole, analyzing of the axial load of sucker rod and lateral loads, and use the lateral deformation of the sucker rod equal annular space between tubing and sucker rod as the basic principle of centralizer allocation, to calculate and analysis of the spacing configuration of the stabilizer. The example analyzed shows that optimization design of sucker rod centralizer spacing has a certain reference value.

\section{Acknowledgements}

The authors would like to thank the Shaanxi Science and Technology Innovation Scheme (2015KTZDGY06-02), Key Problems of Industrial Science and technology of Shaanxi Province (2015GY110), (2016GY-185) and the Local Service Scheme of Shaanxi Province Education Department (15JF027) for financial support.

\section{References}

[1] C. Tan,X. Hu, D. Gao,Three-dimensional calculation of sucker rod centralizer spacing in deviated well, China Petroleum Machinery.25(1997)45-48.

[2] C. Yang, S. Fan,Y. Xu,et al,Side-abrasion of sucker rod string in straight hole , Journal of DaQing Petroleum Institute.24(2004) 68-70.

[3] Z. Huang, X. Luo,H. Wei,et al,Spacing configuration calculation method of sucker rod centralizer in three dimensional hole, Oil Drilling \& Production Technology.32(2010)60-63.

[4] W. Gong, Space form of deviated wells and its practical calculation theory, Natural Gas Industry. 16(1996)35-39.

[5] Z. Gao, Discussion on total angular change rate, Oil Drilling \& Production Technology.7(1985) 35-38. 